\title{
SPATIO-SPECTRAL DECONVOLUTION OF VECTOR VALUED IMAGES USING TOTAL NUCLEAR VARIATION
}

\author{
M. Hidane \\ INSA Centre Val de Loire \\ Laboratoire d'Informatique de l'Université de Tours \\ France
}

\author{
C. Tauber \\ UMRS INSERM U930 \\ Université de Tours \\ France
}

\begin{abstract}
We propose a spatio-spectral deconvolution approach for partial volume correction in $3 \mathrm{D}$ vector-valued imaging. We adopt a variational point of view where we obtain the latent 4D image as a global minimizer of a convex energy function. The prior that we use leverages recent vectorial extensions of the total variation penalty. Specifically, it encourages different channels to share common gradient directions. We discuss why such an approach is appropriate for dynamic PET image deconvolution and derive an efficient algorithm by working in a product-space setting and using the Douglas-Rachford algorithm as a primitive. We use simulations based on a numerical phantom and realistic kinetics of two radioactive tracers to assess the performance of the proposed method.
\end{abstract}

Index Terms - PET, Partial Volume Effect, Deconvolution, Vectorial Total Variation, Douglas-Rachford

\section{INTRODUCTION}

Dynamic positron emission tomography (PET) is a powerful in vivo imaging modality that provides quantitative information on physiological processes. However, the precision of biological parameters derived from dynamic PET data can be both affected by noise and by the relatively poor spatial resolution of PET imaging. The direct consequence of limited spatial resolution is called partial volume effect (PVE), which consists in the inclusion of more than one structure or tissue type in the voxels of the image, and a resolution blurring, called spillover, that contributes to the mixing of image properties among different voxels [1]. This results in a loss of signal for structures partially occupying the point-spreadfunction (PSF) of the "camera". Recently, there has been an increased interest in the development of approaches that consider dynamic PET image as a vector-valued image where each frame can be viewed as a channel in the spectral dimension [2, 3]. Rather than processing each frame independently, such approaches take benefit from the redundancy and complementarity of the globally considered PET data.

In this paper, we propose a non-blind post-reconstruction deconvolution approach for partial volume correction (PVC) of vector-valued images that we apply to dynamic PET data. PVE is modeled by applying the same known PSF to each 3D time frame. Our forward model also incorporates a noise term accounting for both sensing and modeling errors. As the associated inverse problem is known to be severely ill-conditioned [4], we adopt a variational approach in which we recover a latent dynamic 3D volume as a global minimizer of an energy function involving prior and data fidelity terms. More specifically, we propose a convex optimization model involving a spatio-temporal prior term and a quadratic loss. A salient feature of the prior we use is the fact that it yields strong time coupling as it encourages voxels at different time frames to share common gradient directions. We propose an inner-loop free algorithm derived from the Douglas-Rachford primitive [5]. The iterations we obtain involve computations that can be parallelized to a large extent, thus taking advantage of the capabilities of graphics processor units.

\subsection{Related Works}

PVC approaches for static PET images have been considered in different works. The papers [1, 6, 7] provide broad overviews of the methods employed in the field. We note at this point that some of the above cited references propose to perform PVC directly in the sinogram domain, that is, PVC is performed within an image reconstruction algorithm. In this paper, we are primarily interested in post-reconstruction deconvolution approaches for PVC. This setting is of interest whenever one has no direct access to the sinogram of the acquisition. We also note that a second-order deconvolution approach, based on total generalized variation [8] has been proposed for static PET images in [9].

The static deconvolution methods cited above can be applied to each separate time frame in a dynamic setting. As such, they have the traditional marginal processing drawback as they do not leverage time correlations. Spatio-temporal PET reconstruction strategies are reviewed in [10]. To the best of our knowledge, very few works propose truly dynamic post-reconstruction PET PVC methodologies. In [11], the authors propose a weighted least squares deconvolution framework and provide an extensive validation of their method on 
simulated dynamic $\left[{ }^{11} \mathrm{C}\right]$ Raclopride PET data.

By considering a dynamic 3D PET volume consisting of $T$ frames as functions from $\Omega \subset \mathbb{Z}^{3}$ to $\mathbb{R}^{T}$, one can envision adopting vectorial regularization approaches investigated for color and multispectral imaging. In this setting, particular attention has been paid to vectorial extensions of the TV prior [12] (see e.g. [3, 13, 14, 15, 16, 17, 18] and references therein). It is important to note that, when applied to dynamic PET images, that variational approaches that come out form this setting do not involve partial derivatives along the time dimension. Rather, the coupling between the different time frames results from the particular norms applied to the spatial Jacobian of the latent image. It is our goal in the present paper to investigate the potential of this line of works in the specific setting of dynamic post-reconstruction 3D PET deconvolution.

\subsection{Plan of the Paper}

Our contributions are presented in Section 2. Therein, we formulate the discrete forward model relating the latent dynamic 3D PET volume to the observed one. We discuss the particular spatio-temporal regularization term we use and motivate its use for dynamic PET deconvolution. We also discuss relations to some of the above cited works on TV extensions to color and multispectral imaging. We then derive an efficient optimization procedure by rewriting our functional in a product-space setting and deploying the Douglas-Rachford algorithm.

We assess in Section 3 the performance of the proposed method by performing deconvolution experiments on dynamic PET simulations. We carefully explain how these simulations are obtained. We also discuss the choice of the parameters involved in the method and compare the results we get with those obtained by marginal TV deconvolution. We draw final conclusions in Section 4.

\subsection{Notations}

Dynamic 3D images will be written as matrices and denoted by the bold lowercase letters $\mathbf{f}, \mathbf{g} \in \mathbb{R}^{N \times T}$ where $N$ is the number of voxels and $T$ the number of time frames. The particular voxel ordering used is denoted by $\sigma: \sigma(x, y, z)$ is the number of the voxel at coordinates $(x, y, z)$. Linear operators are written as uppercase non-bold letters. Taking the Jacobian of $\mathbf{f}$ leads to a tensor $J \mathbf{f} \in \mathbb{R}^{N \times T \times 3}$. Additional vector fields will be denoted by $\mathbf{p} \in \mathbb{R}^{N \times T \times 3}$. The colon operator allows to take specific slices of a matrix or tensor, for example, $\mathbf{p}_{i, \text { :,: }}$ denotes the $i^{\text {th }} T \times 3$ matrix extracted from the tensor $\mathbf{p}$. The $p$-Schatten norm of a matrix is denoted $\|\cdot\|_{S_{p}}$ and is equal to the $\ell_{p}$ norm of its singular values. The Euclidean norm is simply denoted $\|\cdot\|$. Further notations will be introduced as needed.

\section{SPATIO-SPECTRAL DECONVOLUTION USING TOTAL NUCLEAR VARIATION (TNVD)}

We propose in this section an inverse problem approach to dynamic 3D PET deconvolution that is generic enough to be extended to any vector-valued image deconvolution problem. The latent dynamic volume is obtained as a global minimizer of a convex energy function involving a regularization term that couples all time frames. We first describe the forward model we work with.

\subsection{The Forward Model}

We consider the following discrete model

$$
\mathbf{g}=H \mathbf{f}+\boldsymbol{\eta} \in \mathbb{R}^{N \times T},
$$

where $H$ denotes a linear blurring operator and $\boldsymbol{\eta}$ is an error term. The operator $H$ is time-separable, meaning that it applies the same blurring to all time frames. We note at this point that the spatial resolution in PET imaging may vary along the radial direction, leading to a space-variant blur $H$. Since our primary focus in this paper is to assess the relative performance gain obtained when considering temporal coupling, we adopt a simplified space-invariant model for $H$. We further work with circular boundary conditions, leading to $H$ being diagonalized by the (three-dimensional) Fourier transform.

In the projection space, the acquired measurements are usually contaminated by Poisson noise. After linear reconstruction, and for a sufficiently large number of iterations, the noise term can reasonably be approximated by a white Gaussian one.

\subsection{The Energy}

Let $(x, y, z)$ denote the coordinates of a voxel $i=\sigma(x, y, z)$ not belonging to the boundary of the volume. For a dynamic volume $\mathbf{f} \in \mathbb{R}^{N \times T}$, let $J \mathbf{f} \in \mathbb{R}^{N \times T \times 3}$ denote its Jacobian, given by:

$$
\begin{aligned}
& (J \mathbf{f})_{\sigma(x, y, z), j, 1}=\mathbf{f}_{\sigma(x+1, y, z), j}-\mathbf{f}_{\sigma(x, y, z), j}, \\
& (J \mathbf{f})_{\sigma(x, y, z), j, 2}=\mathbf{f}_{\sigma(x, y+1, z), j}-\mathbf{f}_{\sigma(x, y, z), j}, \\
& (J \mathbf{f})_{\sigma(x, y, z), j, 3}=\mathbf{f}_{\sigma(x, y, z+1), j}-\mathbf{f}_{\sigma(x, y, z), j} .
\end{aligned}
$$

We assume circular boundary conditions for the definition of $J$ so that $J$ is also diagonalized by the (three-dimensional) Fourier transform.

The different vectorial TV extensions proposed in $[15,16$, $17,18]$ are obtained by applying specific norms to the Jacobian defined in (2). Of particular interest to our present setting are the formulations involving the Schatten p-norms of the Jacobian at each voxel:

$$
R_{p}(\mathbf{f}):=\|J \mathbf{f}\|_{1, S_{p}}:=\sum_{i=1}^{N}\left\|(J \mathbf{f})_{i,:,:}\right\|_{S_{p}} .
$$


The use of $R_{p}$ as a prior, together with the white Gaussian noise assumption lead to consider the following convex problem:

$$
\underset{\mathbf{x} \in \mathbb{R}^{N \times T}}{\operatorname{Minimize}} R_{p}(\mathbf{x})+\frac{\lambda}{2}\|H \mathbf{x}-\mathbf{g}\|^{2}
$$

The particular choice $p=1$ corresponds to total nuclear variation and has been extensively considered in [16] in the context of color image denoising, namely when $H$ is the identity. An important feature of the prior $R_{1}$ is the fact that it can be seen as a convex relaxation of the sum of the rank of the Jacobian at each voxel [19]. This prior is also encapsulated in those proposed in [17] and [18] and is used in [20] for joint spectral CT reconstruction.

When applied to dynamic PET data, the regularizer $R_{1}$ thus encourages different time frames to share common gradient directions. We argue that this salient feature is particularly useful for dynamic PET imaging for the following reason. The contrast between adjacent regions depends on several factors. In the early frames, it relies strongly on the perfusion of the tissues, while in the late frames, it relies more on the specific binding of the radiotracer. Therefore the contrast between regions often changes across the different frames, and can sometimes become opposite. However, on a given edge voxel the gradient directions are aligned across frames, which makes the $R_{1}$ regularizer very appropriate for dynamic PET image processing. We note that $R_{1}$ still allows for large gradient direction changes at specific voxels since the local $\left\|(J \mathbf{f})_{i,:,:}\right\|_{S_{1}}$ norms are spatially aggregated through the robust $\ell_{1}$ norm.

\subsection{Optimization}

Following the previous discussion, we tackle the dynamic PET deconvolution problem by solving the convex problem

$$
\underset{\mathbf{x} \in \mathbb{R}^{N \times T}}{\operatorname{Minimize}}\|J(\mathbf{x})\|_{1, S_{1}}+\frac{\lambda}{2}\|H \mathbf{x}-\mathbf{g}\|^{2} .
$$

To carry out the minimization we introduce an auxiliary variable and rewrite (5) in the form

$$
\underset{(\mathbf{x}, \mathbf{p}) \in \mathbb{R}^{N \times T} \times \mathbb{R}^{N \times T \times 3}}{\operatorname{Minimize}}\|\mathbf{p}\|_{1, S_{1}}+\frac{\lambda}{2}\|H \mathbf{x}-\mathbf{g}\|^{2}+\iota_{C}(\mathbf{x}, \mathbf{p}),
$$

where $\iota_{K}$ denotes the indicator function of a closed convex set $K$ and is given by $\iota_{K}(x)=0$ if $x \in K$ and $\iota_{K}(x)=\infty$ otherwise, and $C=\left\{(\mathbf{x}, J \mathbf{x}), \mathbf{x} \in \mathbb{R}^{N \times T}\right\}$.

We apply the Douglas-Rachford algorithm [5] with the following identifications: $F_{1}(\mathbf{x}, \mathbf{p})=\|\mathbf{p}\|_{1, S_{1}}+\frac{\lambda}{2}\|H \mathbf{x}-\mathbf{g}\|^{2}$ and $F_{2}(\mathbf{x}, \mathbf{p})=\iota_{C}(\mathbf{x}, \mathbf{p})$. Through the iterations, the algorithm alternates between computing the proximity operator of $F_{1}$ and projecting on the constraint subspace $C$. Since $F_{1}$ is separable across $\mathbf{x}$ and $\mathbf{p}$, so is its proximity operator.

The different computational steps are summarized in Algorithm 1 . We note that the $x_{1}^{[l]}$ update is time-separable and can be efficiently implemented with direct and inverse 3D Fourier transforms. The same applies for the $\mathbf{x}_{2}^{[l]}$ update rule. Finally, the $\mathbf{p}_{2}^{[l]}$ update is space-separable and involves the singular value thresholding (SVT) function defined for all matrices $\mathbf{A}$ and $\nu>0$ by $\operatorname{SVT}_{\nu}(\mathbf{A})=\mathbf{U} \max (\boldsymbol{\Sigma}-\nu \mathbf{l}, 0) \mathbf{V}^{*}$, where $\mathbf{A}=\mathbf{U} \boldsymbol{\Sigma} \mathbf{V}^{*}$ is a singular value decomposition of $\mathbf{A}$ and max denotes the entry-wise maximum.

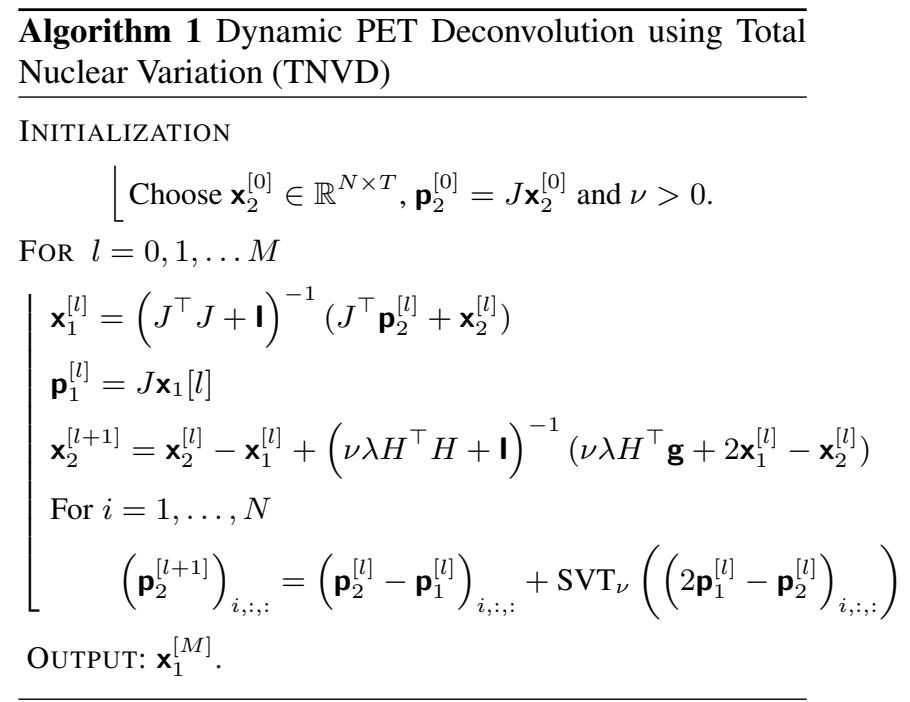

\section{EXPERIMENTS}

\subsection{Image Simulation}

To evaluate the accuracy of the proposed vector-valued deconvolution approach, we used simulated dynamic PET data. We considered the Zubal head phantom as a voxelized brain source [21]. Two radiotracers were considered: [18F]LBT999 and [18F]FDG. For each region of the brain, typical kinetics were derived from clinical PET data by calculating an average time activity curve using a registered anatomical atlas. The ground truth was first downsampled to match the voxel size of a PET "camera". The image was convolved with a gaussian kernel approximating an isotropic 4mm PSF. Finally an additive Gaussian noise with standard deviation equal to 5 was added to account for the propagation of sensing errors in the reconstructed dynamic PET volume.

\subsection{Parameters and Comparisons}

The method proposed in Algorithm 1 has three parameters: $\lambda$ (the regularization parameter), $\nu$ (the rate parameter in Douglas-Rachford) and $M$ the number of iterations. The value of $\lambda$ has been set empirically. The value $\lambda=0.5$ has been chosen for the [18F]LBT-999 tracer while the value $\lambda=0.3$ has been chosen for the $[18 \mathrm{~F}] \mathrm{FDG}$ tracer. Regarding, the parameter $\nu$, we note that while the convergence of Algorithm 1 is guaranteed for every $\nu>0$, in practice, the choice 
Table 1. PFOM and SSIM for two deconvolution simulations on the same phantom using two different radioactive tracers. The first two rows correspond to PFOM and SSIM values obtained with marginal TV deconvolution. The last two rows correspond to the results with the proposed TNVD spatio-temporal approach. Each column corresponds to a particular time frame. From a total of 20 frames, only odd numbered ones are shown.

Simulation 1 ([18F]LBT-999)

Simulation 2 ([18F]FDG)

\begin{tabular}{|c|c|c|c|c|c|c|c|c|c|c|c|c|c|c|c|c|c|c|c|c|c|}
\hline \multirow{2}{*}{ MTVD } & PFOM & 0.426 & 0.520 & 0.558 & 0.556 & 0.534 & 0.487 & 0.451 & 0.494 & 0.424 & 0.450 & 0.417 & 0.569 & 0.576 & 0.591 & 0.608 & 0.620 & 0.630 & 0.655 & 0.667 & 0.678 \\
\hline & SSIM & 0.358 & 0.246 & 0.261 & 0.264 & 0.258 & 0.250 & 0.242 & 0.245 & 0.235 & 0.231 & 0.688 & 0.688 & 0.679 & 0.686 & 0.693 & 0.695 & 0.702 & 0.710 & 0.715 & 0.718 \\
\hline \multirow[t]{2}{*}{ TNVD } & PFOM & 0.238 & 0.671 & 0.675 & 0.679 & 0.681 & 0.665 & 0.653 & 0.628 & 0.631 & 0.608 & 0.425 & 0.619 & 0.629 & 0.651 & 0.667 & 0.677 & 0.681 & 0.701 & 0.701 & 0.712 \\
\hline & SSIM & 0.690 & 0.667 & 0.635 & 0.629 & 0.628 & 0.633 & 0.637 & 0.645 & 0.660 & 0.654 & 0.715 & 0.743 & 0.738 & 0.745 & 0.747 & 0.750 & 0.755 & 0.757 & 0.759 & 0.756 \\
\hline
\end{tabular}

of $\nu$ has a dramatic effect on the energy profile. The value $\nu=10$ has been used for the numerical simulations reported in the present paper and leads to a stable energy profile with a number of iterations, $M$, varying between 20 and 30 .

When the proposed method is applied to a scalar volume $(T=1)$, one recovers the classical TV deconvolution approach. Since our primary goal is to assess the impact of coupled versus marginal processing, we compare our method with marginal TV deconvolution (MTVD), that is TV deconvolution applied to each time frame separately.

The results obtained with the MTVD and TNVD approaches were assessed both in image quality using the SSIM index, and in edge sharpening using Pratt's figure of merit (PFOM) after applying a Canny edge detector.

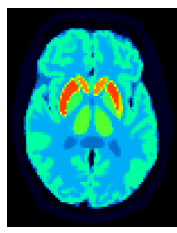

(a)

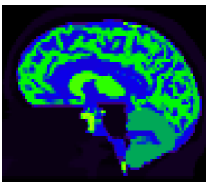

(e)

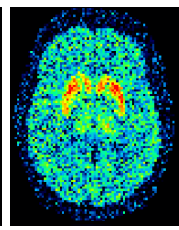

(b)

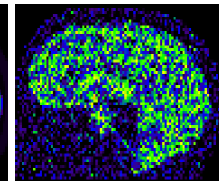

(f)

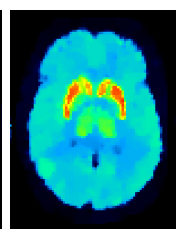

(c)

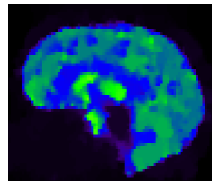

(g)

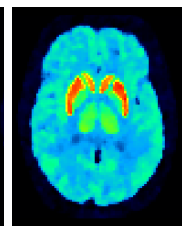

(d)

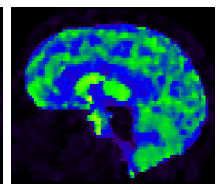

(h)
Fig. 1. Sample views of results obtained on simulated vectorvalued PET images. Top row: Results displayed in transverse plane, a) Ground truth, b) Simulated image, c) MTVD, d) TNVD. Bottom row: Results displayed in sagittal plane, e) Ground truth, f) Simulated image, g) MTVD, h) TNVD.

\subsection{Results}

Representative results are presented in Figure 1. All images of a given row are scaled to a common colorscale. In both rows, the first column contains the ground truth that was used both for image simulation (second column) and for quantitative evaluation of MTVD (third column) and our proposed TNVD (fourth column). A visual comparison suggests that, compared to the simulated image, both methods have strongly fil- tered the noise and increased the signal-to-noise ratio. However, it can be noted that the borders between gray and white matter have almost disappeared with MTVD. Moreover, the thalamic nuclei is less sharp and the radiotracer concentration appears lower in the striatum. On the contrary, the edges between regions of interest appear sharper with TNVD and more precisely located. The quantitative bias in the striatum has been partially compensated by TNVD.

Quantitative results are presented in Table 1. For each simulation, we present the values obtained over different frames of the dynamic PET images, both for MTVD and TNVD. For both simulations, both the PFOM and the SSIM were significantly increased by TNVD compared to MTVD, with the latter obtaining the highest score only once. Such results suggest that the proposed approach can effectively enhance the quality of dynamic PET images by both sharpening the edges between kinetic regions and reducing the quantitative bias.

\section{CONCLUSION}

We have introduced a post-reconstruction spatio-temporal deconvolution approach for dynamic PET PVC. Adopting a variational point of view, we have obtained the latent dynamic 3D volume as a global minimizer of a convex energy function. The salient feature of the proposed model is the fact that it encourages different time frames to share common gradient directions. This is accomplished by penalizing the nuclear norm of the Jacobian at each voxel and then spatially aggregating these local penalties with the $\ell_{1}$ norm. We have conducted numerical experiments on one physical phantom and two radioactive tracers and showed that, in terms of structural similarity and Pratt's figure of merit, the proposed method outperforms marginal TV deconvolution.

Future work will include more thorough experimental validation, in particular on GATE [22] simulations as well as on real dynamic PET imaging. This validation should also include issues related to quantitative kinetic analysis in order to be useful in a clinical context.

It should be noted that the proposed approach is very generic and that besides dynamic PET imaging, it can be used for the noise filtering and deconvolution of many other vector-valued imaging modalities. 


\section{REFERENCES}

[1] Marine Soret, Stephen L Bacharach, and Irene Buvat, "Partial-volume effect in pet tumor imaging," Journal of Nuclear Medicine, vol. 48, no. 6, pp. 932-945, 2007.

[2] C Tauber, S Stute, M Chau, P Spiteri, S Chalon, D Guilloteau, and I Buvat, "Spatio-temporal diffusion of dynamic pet images," Physics in medicine and biology (IF 2.92), vol. 56, no. 20, pp. 6583, 2011.

[3] V. Jaouen, P. Gonzalez, S. Stute, D. Guilloteau, S. Chalon, I. Buvat, and C. Tauber, "Variational segmentation of vector-valued images with gradient vector flow," IEEE Transactions in Image Processing (IF 3.11), vol. 23, no. 11, pp. 4773-4785, 2014.

[4] Mario Bertero and Patrizia Boccacci, Introduction to inverse problems in imaging, CRC press, 2010.

[5] Patrick L Combettes and Jean-Christophe Pesquet, "A Douglas-Rachford splitting approach to nonsmooth convex variational signal recovery," Selected Topics in Signal Processing, IEEE Journal of, vol. 1, no. 4, pp. 564-574, 2007.

[6] Kjell Erlandsson, Irene Buvat, P Hendrik Pretorius, Benjamin A Thomas, and Brian F Hutton, "A review of partial volume correction techniques for emission tomography and their applications in neurology, cardiology and oncology," Physics in medicine and biology, vol. 57, no. 21, pp. R119, 2012.

[7] Arman Rahmim, Jinyi Qi, and Vesna Sossi, "Resolution modeling in pet imaging: theory, practice, benefits, and pitfalls," Medical physics, vol. 40, no. 6, 2013.

[8] Kristian Bredies, Karl Kunisch, and Thomas Pock, "Total generalized variation," SIAM Journal on Imaging Sciences, vol. 3, no. 3, pp. 492-526, 2010.

[9] Stéphanie Guérit, Laurent Jacques, Benoît Macq, and John A Lee, "Post-reconstruction deconvolution of pet images by total generalized variation regularization," in Signal Processing Conference (EUSIPCO), 2015 23rd European. IEEE, 2015, pp. 629-633.

[10] Arman Rahmim, Jing Tang, and Habib Zaidi, "Fourdimensional (4d) image reconstruction strategies in dynamic pet: Beyond conventional independent frame reconstruction," Medical physics, vol. 36, no. 8, pp. 36543670, 2009.

[11] Anthonin Reilhac, Arnaud Charil, Catriona Wimberley, Georgios Angelis, Hasar Hamze, Paul Callaghan, Marie-Paule Garcia, Frederic Boisson, Will Ryder, Steven R Meikle, et al., " $4 \mathrm{~d}$ pet iterative deconvolution with spatiotemporal regularization for quantitative dynamic pet imaging," NeuroImage, vol. 118, pp. 484493, 2015.

[12] L. Rudin, S. Osher, and E. Fatemi, "Nonlinear total variation based noise removal algorithms," Physica $D$, vol. 60, no. 1-4, pp. 259-268, 1992.

[13] Peter Blomgren and Tony F Chan, "Color tv: Total variation methods for restoration of vector-valued images," IEEE transactions on image processing, vol. 7, no. 3, pp. 304-309, 1998.

[14] Xavier Bresson and Tony F Chan, "Fast dual minimization of the vectorial total variation norm and applications to color image processing," Inverse problems and imaging, vol. 2, no. 4, pp. 455-484, 2008.

[15] Bastian Goldluecke, Evgeny Strekalovskiy, and Daniel Cremers, "The natural vectorial total variation which arises from geometric measure theory," SIAM Journal on Imaging Sciences, vol. 5, no. 2, pp. 537-563, 2012.

[16] Kevin M Holt, "Total nuclear variation and jacobian extensions of total variation for vector fields," IEEE Transactions on Image Processing, vol. 23, no. 9, pp. 3975-3989, 2014.

[17] Stamatios Lefkimmiatis, Anastasios Roussos, Petros Maragos, and Michael Unser, "Structure tensor total variation," SIAM Journal on Imaging Sciences, vol. 8, no. 2, pp. 1090-1122, 2015.

[18] Juan Duran, Michael Moeller, Catalina Sbert, and Daniel Cremers, "Collaborative total variation: a general framework for vectorial tv models," SIAM Journal on Imaging Sciences, vol. 9, no. 1, pp. 116-151, 2016.

[19] Benjamin Recht, Maryam Fazel, and Pablo A Parrilo, "Guaranteed minimum-rank solutions of linear matrix equations via nuclear norm minimization," SIAM review, vol. 52, no. 3, pp. 471-501, 2010.

[20] David S Rigie and Patrick J La Rivière, "Joint reconstruction of multi-channel, spectral ct data via constrained total nuclear variation minimization," Physics in medicine and biology, vol. 60, no. 5, pp. 1741, 2015.

[21] I. George Zubal, Charles R. Harrell, Eileen O. Smith, Zachary Rattner, Gene Gindi, and Paul B. Hoffer, "Computerized three-dimensional segmented human anatomy," Medical Physics, vol. 21, no. 2, pp. 299-302, feb 1994.

[22] S Jan, G Santin, D Strul, Steven Staelens, K Assie, D Autret, S Avner, R Barbier, M Bardies, PM Bloomfield, et al., "Gate: a simulation toolkit for pet and spect," Physics in medicine and biology, vol. 49, no. 19, pp. 4543, 2004. 